\title{
How does editing the genome improve targeting DNA and RNA for cerebellar ataxias?
}

\author{
Jordi Gandini ${ }^{1}$ \& Mario Manto*,1,2 \\ ${ }^{1}$ Cerebellar Ataxia Unit, Department of Neurology, CHU-Charleroi, Charleroi, Belgium \\ ${ }^{2}$ Department of Neuroscience, University of Mons, Mons, Belgium \\ *Author for correspondence: mmanto@ulb.ac.be
}

First draft submitted: 6 October 2019; Accepted for publication: 18 October 2019; Published online: 14 January 2020

Keywords: ataxias • cerebellum • DNA • RNA • therapies

\section{The heterogeneity of cerebellar disorders}

Cerebellar ataxias (CAs) represent a group of disabling disorders affecting all ages and represented worldwide. CAs are highly heterogeneous disorders in terms of phenotypes and genotypes [1]. Patients exhibit pure cerebellar deficits or complex combinations of neurological signs related to the central nervous system (cerebellum, cerebral cortex, basal ganglia, brainstem, cranial nerves, spinal cord) and/or peripheral nervous system involvement. Clinically, patients show, in particular, oculomotor deficits (nystagmus, oculomotor dysmetria), motor symptoms affecting speech (dysarthria), limbs and gait (dysmetria, kinetic tremor, unsteady gait) as well as a wide range of cognitive impairments.

\section{Recent progress in the characterization of CAs}

The multimodal development of next-generation sequencing, novel neuroimaging tools such as tractography and functional magnetic resonance imaging (fMRI), relevant cellular models and dedicated animal models have not only extended our appraisal of cerebellar disorders from the clinical standpoint but have also improved drastically the identification of the molecular/cellular mechanisms leading to CAs encountered in daily practice [2]. There is also substantial progress in the field of biomarkers to identify the premanifest stage and to monitor disease progression, such asvolumetry of the cerebellum and spinal cord, fMRI assessment of brain networks, magnetic resonance spectroscopy, oculomotor biomarkers, quantificative metric tools, neurofilament light chain -Nfl- in the serum [3,4]. Neuroimaging biomarkers are particularly attractive. Not only the atrophy of the brain evolves in specific patterns in several CAs, but it is also possible to detect abnormalities at a premanifest stage. fMRI pattern at rest or during tasks is also a matter of investigation.

\section{Polyglutaminopathies}

Nucleotide repeat disorders (NRDs) represent debilitating CAs with a recessive or dominant inheritance [5]. NRDs are characterized by tandem copies of a specific DNA sequence in genes, with repeat expansions in one or both alleles and repeat instability leading to anticipation [5]. Expanded sequences affect both coding and noncoding regions. Genetic instability and inverse correlation between the size of expanded repeats and severity of the disease are key-features. Disease onset is usually between 20 and 45 years, with a phenomenon of anticipation in successive generations. The most common NRD are trinucleotide repeat sequences, but repeats also include tetra, penta or hexanucleotide repeats. Several NRDs, such as spinocerebellar ataxia (SCA) $1 / 2 / 3 / 6 / 7 / 17$, show an expansion of CAG repeats resulting in toxic RNA and/or polyglutamine tracts (we will not discuss Huntington's disease and myotonic dystrophy here) [6]. The expanded polyQ (Qs: glutamine) sequence generates an insoluble protein aggregate occurring especially in the neurons of the cerebellum, perturbing key-cellular processes and leading to neuronal death (hence the neurodegenerative feature). The size of the CAG repeat dictates the severity of the phenotype. The expanded CAG repeats result in gain-of-function disorders with corresponding pathological proteins called ataxins. By comparison, Friedreich ataxia (FRDA) and fragile-X syndrome represent exemplative 
cases of NRDs with a loss-of-function pathogenesis. Most FRDA patients show an expansion of the GAA*TCC repeat in the frataxin gene, causing deficits in frataxin, hence the objective to increase the amounts of mRNA and/or frataxin level [7]. Fragile-X syndrome is due to a CGG*CCG of the FMR1 gene. When the expansion overpasses 200 repeats, the gene is silent and the protein FMRP is reduced. A number of copies between 55 and 200 (premutated alleles) is a risk factor for FXTAS. Noteworthy, these patients have an increased transcription of FMR1 mRNA. However, FMRP translation is impaired. Other mechanisms of CAs include mitochondrial dysfunction, autophagy and channelopathies.

\section{Therapies of CAs}

Unfortunately, most of the symptomatic therapies such as conventional oral medications aiming to restore defects in neurotransmitters (mainly GABA, glutamate, serotonin, acetylcholine in the cerebellum machinery) or regular rehabilitation/motor training methods currently administered have failed to revert the fatal evolution of NRT [8]. Noninvasive stimulation tools aiming to modulate the excitability of the cerebellar cortex are being assessed. We also miss an effective approach to reach a real prevention of clinical deficits in most genetic ataxias. Exceptions to the rule in the field of CAs include immune ataxias for which effective therapies are available and are effective when the cerebellar reserve is still sufficient [9]. In other words, the therapies improve the clinical deficits if administered at an early stage when the neuronal loss and inflammatory reaction are mild or moderate.

Therapeutics targeting DNA and RNA represent a revolution in the management of genetic disorders of the cerebellum. These novel tools include, in particular, oligonucleotides, which are biologically active compounds. A typical example is 5-methyl-cytosine which targets RNA and increases its stability. Antisense oligonucleotides (ASOs) have a RNA-like structure. They are short synthetic strings of nucleic acids with a length of 10-50 nucleotides. In gain-of-function disorders, the main objective is to decrease the mRNA contents by use of singlestranded ASOs, by blocking translation or by a direct silencing effect [10]. ASOs bind complementary mRNA using Watson-Crick hybridization, leading to RNase H enzymes recruitement [11]. It has been demonstrated in the mouse model of SCA2 that intrathecal administration of ASOs decreases the levels of the mutated protein and improves the firing of Purkinje neurons in the cerebellar cortex as well as motor coordination [12]. Mice receiving ASOs show a slower progression of motor deficits and longer survival [13]. Similar findings have been reported in the SCA3 mouse model [14]. ASOs reduce to $50 \%$ the pathological ataxin-3 in the cerebellum, diencephalon, forebrain and cervical spinal cord without microgliosis or astrogliosis [15]. Reducing the insoluble ataxin-3 is beneficial on the pathogenicity [16]. Allele-specific ASOs are being studied. The ASOs would preferentially bind to the longer repeat. Nevertheless, trying to down-regulate the mutant allele only may not be interesting for all NRDs and therefore both alleles might be targeted, keeping in mind that the proteins under consideration do play important physiological roles. Other techniques such as exon skipping try to exclude the exon with the CAG repeat from the transcript.

The aim of RNA interference (RNAi) is to block the expression of mutated polyglutamine-proteins resulting from expanded polyglutamine mutations. Synthetic small interfering RNA (siRNAs) and short hairpin RNA (shRNAs) regulate the RNAi process of target mRNA enzymatic cleavage. RNAi decreases the levels of ataxin-7 in the mouse model of SCA7, with significant improvement of the ataxic phenotype [17]. The cerebellar molecular layer thinning is blocked and nuclear inclusions, which represent a hallmark of this group of diseases, are reduced. One of the effects of polyQ proteins is to impair transcription regulation and therefore attempts are ongoing to revert this mechanism using histone deacetylases such as valproic acid. This molecule upregulates gene transcription through hyperacetylation [18]. In FRDA, delivering the mRNA is also currently investigated. dsRNA (anti-trinucleotide GAA duplex RNAs) activates the FXN gene expression. Single-stranded siRNAs which combine the strengths of dsRNA and ASOs are also potential therapies [19].

Nascent polypeptide associated complex, a ribosome-associated protein biogenesis factor, suppresses the aggregation of polyglutamine (polyQ)-expanded proteins associated with several types of SCAs [20]. Nascent polypeptide associated complex exerts a chaperone activity. This molecule represents a promising therapy for SCAs when the goal is to suppress aggregation and proteotoxicity of mutant polyQ-expanded proteins.

\section{Conclusion}

In conclusion, nucleic acid-based therapeutic strategies have a huge potential for NRDs. The research community has moved to a phase of identification of the mechanisms of ataxias to a real reorganization of the pathological DNA-RNA machinery. There is a great hope that these novel therapeutic strategies will be used very soon to block 
the onset of symptoms in CAs, already at the premanifest stage when the cerebellar reserve is high. Safety and tolerability profiles are encouraging. Genome editing of CAs has entered in reality.

\section{Author contributions}

J Gandini \& M Manto drafted the manuscript. J Gandini \& M Manto revised the manuscript and agreed on the final version.

\section{Financial \& competing interests disclosure}

M Manto is the Chief Editor of The Cerebellum, the Chief Editor of Cerebellum and Ataxias, the Deputy Editor of the Journal of NeuroEngineeing and Rehabilitation and the Editor of Contemporary Clinical Neurosciences. He has received royalties from Cambridge University Press, Springer, Lavoisier Medecine, Elsevier, Morgan and Claypool. The authors have no other relevant affiliations or financial involvement with any organization or entity with a financial interest in or financial conflict with the subject matter or materials discussed in the manuscript apart from those disclosed.

No writing assistance was utilized in the production of this manuscript.

\section{Open access}

This work is licensed under the Attribution-NonCommercial-NoDerivatives 4.0 Unported License. To view a copy of this license, visit http://creativecommons.org/licenses/by-nc-nd/4.0/

\section{References}

Papers of special note have been highlighted as: $\bullet$ of interest; $\bullet \bullet$ of considerable interest

1. Kuo SH. Ataxia. Continuum 25(4), 1036-1054 (2019).

- Reviews the current status of ataxia.

2. Synofzik M, Puccio H, Mochel F, Schöls L. Autosomal recessive cerebellar ataxias: paving the way toward targeted molecular therapies. Neuron 101(4), 560-583 (2019).

-. An outstanding paper on the pathogenesis of recessive ataxias.

3. Deelchand DK, Joers JM, Ravishankar A et al. Sensitivity of volumetric magnetic resonance imaging and magnetic resonance spectroscopy to progression of spinocerebellar ataxia type 1. Mov. Disord. Clin. Pract. 6(7), 549-558 (2019).

4. Shirai S, Yabe I, Takahashi-Iwata I et al. The responsiveness of triaxial accelerometer measurement of gait ataxia is higher than that of the scale for the assessment and rating of ataxia in the early stages of spinocerebellar degeneration. Cerebellum 18(4), 721-730 (2019).

5. Zain R, Smith CIE. Targeted oligonucleotides for treating neurodegenerative tandem repeat diseases. Neurotherapeutics 16(2), 248-262 (2019).

6. Klockgether T, Mariotti C, Paulson HL. Spinocerebellar ataxia. Nat. Rev. Dis. Primers 5(1), 24 (2019).

7. Gottesfeld JM. Molecular mechanisms and therapeutics for the GAA.TTC expansion disease Friedreich ataxia. Neurotherapeutics doi:10.1007/s13311-019-00764-x (2019) (Epub ahead of print).

8. Perlman S, Boltshauser E. Drug treatment. Handb. Clin. Neurol. 155, 371-377 (2018).

- Reviews the current therapies of cerebellar disorders.

9. Mitoma H, Manto M, Hampe CS. Immune-mediated cerebellar ataxias: practical guidelines and therapeutic challenges. Curr. Neuropharmacol. 17(1), 33-58 (2019).

10. Moore LR, Rajpal G, Dillingham IT et al. Evaluation of antisense oligonucleotides targeting ATXN3 in SCA3 mouse models. Mol. Ther. Nucleic Acids 7, 200-210 (2017).

11. Sullivan R, Yau WY, O’Connor E et al. Spinocerebellar ataxia: an update. J. Neurol. 266, 533-544 (2019).

12. Scoles DR, Meera P, Schneider MD et al. Antisense oligonucleotide therapy for spinocerebellar ataxia type 2. Nature 544(7650), 362-366 (2017).

- An excellent report on the effects of antisense oligonucleotides for spinocerebellar ataxia type 2 .

13. Becker LA, Huang B, Bieri G et al. Therapeutic reduction of ataxin-2 extends lifespan and reduces pathology in TDP-43 mice. Nature 544(7650), 367-371 (2017).

14. McLoughlin HS, Moore LR, Chopra R et al. Oligonucleotide therapy mitigates disease in spinocerebellar ataxia type 3 mice. Ann. Neurol. 84(1), 64-77 (2018).

15. Moore LR, Rajpal G, Dillingham IT et al. Evaluation of antisense oligonucleotides targeting ATXN3 in SCA3 mouse models. Mol. Ther. Nucleic Acids 7, 200-210 (2017).

16. Toonen LJA, Rigo F, van Attikum $\mathrm{H}$ et al. Antisense oligonucleotide-mediated removal of the polyglutamine repeat in spinocerebellar ataxia type 3 mice. Mol. Ther. Nucleic Acids 8, 232-242 (2017). 
17. Ramachandran PS, Boudreau RL, Schaefer KA et al. Nonallele specific silencing of ataxin-7 improves disease phenotypes in a mouse model of SCA7. Mol. Ther. 22(9), 1635-1642 (2014).

18. Lin XP, Feng L, Xie CG et al. Valproic acid attenuates the suppression of acetyl histone H3 and CREB activity in an inducible cell model of Machado-Joseph disease. Int. J. Dev. Neurosci. 38, 17-22 (2014).

19. Shen X, Kilikevicius A, O'Reilly D et al. Activating frataxin expression by single-stranded siRNAs targeting the GAA repeat expansion. Bioorg. Med. Chem. Lett. 28(17), 2850-2855 (2018).

20. Shen K, Gamerdinger M, Chan R et al. Dual role of ribosome-binding domain of NAC as a potent suppressor of protein aggregation and aging-related proteinopathies. Mol. Cell 74(4), 729-741 (2019). 